\title{
The effectiveness of a nurse-led illness perception intervention in COPD patients: a cluster randomised trial in primary care
}

\author{
Saskia W.M. Weldam ${ }^{1}$, Marieke J. Schuurmans ${ }^{2}$, Pieter Zanen ${ }^{1}$, \\ Monique J.W.M. Heijmans ${ }^{3}$, Alfred P.E. Sachs ${ }^{4}$ and Jan-Willem J. Lammers ${ }^{1}$
}

Affiliations: ${ }^{1}$ Dept of Respiratory Diseases, Division of Heart and Lungs, University Medical Center Utrecht, Utrecht University, Utrecht, the Netherlands. ${ }^{2}$ Dept of Nursing Science, Julius Center for Health Sciences and Primary Care, University Medical Center Utrecht, Utrecht University, Utrecht, the Netherlands. ${ }^{3}$ Netherlands Institute for Health Services Research (Nivel), Utrecht, the Netherlands. ${ }^{4}$ Julius Center for Health Sciences and Primary Care, University Medical Centre Utrecht, Utrecht University, Utrecht, the Netherlands.

Correspondence: Saskia W.M. Weldam, University Medical Center Utrecht, HP E03.511, PO Box 85500, 3508 GA, Utrecht, The Netherlands. E-mail: S.Weldamवumcutrecht.nl

ABSTRACT The new COPD-GRIP (Chronic Obstructive Pulmonary Disease - Guidance, Research on Illness Perception) intervention translates evidence regarding illness perceptions and health-related quality of life (HRQoL) into a nurse intervention to guide COPD patients and to improve health outcomes. It describes how to assess and discuss illness perceptions in a structured way. This study aimed to assess the effectiveness of the intervention in primary care.

A cluster randomised controlled trial was conducted within 30 general practices and five home-care centres, including 204 COPD patients. 103 patients were randomly assigned to the intervention group and 101 patients to the usual-care group. To assess differences, repeated multilevel linear mixed modelling analyses were used. Primary outcome was change in health status on the Clinical COPD Questionnaire (CCQ) at 9 months. Secondary outcomes were HRQoL, daily activities, health education impact and changes in illness perceptions.

There was no significant difference between the groups in the CCQ at 9 months. We found a significant increase in health-directed behaviour at 6 weeks $(p=0.024)$ and in personal control $(p=0.005)$ at 9 months in favour of the intervention group.

The COPD-GRIP intervention, practised by nurses, did not improve health status in COPD patients in primary care. However, the intervention has benefits in improving the ability to control the disease and health-related behaviours in the short term. Therefore, taking illness perceptions into account when stimulating healthy behaviours in COPD patients should be considered. Further study on influencing the health status and HRQoL is needed.

@ERSpublications

COPD illness perception intervention improved health behaviours in the short term, but did not improve health status http://ow.ly/1VSw30fQQjN

Cite this article as: Weldam SWM, Schuurmans MJ, Zanen P, et al. The effectiveness of a nurse-led illness perception intervention in COPD patients: a cluster randomised trial in primary care. ERJ Open Res 2017; 3: 00115-2016 [https://doi.org/10.1183/23120541.00115-2016].

Received: Oct 132016 | Accepted after revision: Sept 302017

This study is registered at the Netherlands Trial Register with identifier number NTR 3945

Support statement: This study is funded by Partners in Care Solutions for COPD (PICASSO). PICASSO had no role in the design, collection, analysis and interpretation of data; in the writing of the report; or in the decision to submit the article for publication.

Conflict of interest: Disclosures can be found alongside this article at openres.ersjournals.com

Copyright $\odot$ ERS 2017. This article is open access and distributed under the terms of the Creative Commons Attribution Non-Commercial Licence 4.0. 


\section{Introduction}

Chronic obstructive pulmonary disease (COPD) is a common chronic disease characterised by respiratory complaints such as dyspnoea and cough, pulmonary function abnormalities mainly due to nonreversible airway obstruction, and limitations in daily life [1]. It is estimated that $\sim 328$ million people worldwide have COPD [2], and their number is still increasing, leading to high social and economic burdens [2, 3].

Three major challenges of COPD care are to reduce the impact of symptoms, to improve health status and health-related quality of life (HRQoL) and to guide patients in their daily life with the consequences of the disease $[4,5]$. In the Netherlands, as well as in other countries, the care for COPD patients has increasingly moved from hospital to primary care settings $[6,7]$. In this context, practice nurses in primary care play an important role in the integrated care of COPD patients $[6,8,9]$.

Although the clinical diagnosis of COPD is usually based upon the degree of airflow limitation (i.e. the decrease in the forced expiratory volume in $1 \mathrm{~s}(\mathrm{FEV} 1)$ and the FEV1/forced vital capacity ratio), individual differences in HRQoL can only be partly explained by these clinical parameters [10]. To understand and explain individual differences in presentation and outcomes of COPD among people with the same (medical) disease severity, other models in addition to biomedical perspectives are increasingly used, for example the common sense model by Leventhal et al. [11]. This model provides a dynamic framework for describing and understanding the processes involved in managing health threats [11, 12]. The model assumes that, when confronted with health threats, people create personal ideas about their illness and treatment in order to make sense of and manage their situation. These ideas, which often do not match medical views, influence coping behaviours and outcomes [11, 12]. These ideas include beliefs about consequences, the ability to control the disease, timeline of the disease, the extent to which the treatment helps in controlling the disease, influence of symptoms, understanding the disease, concerns and emotional responses to the disease $[11,12]$.

Extensive reviews have shown that illness perceptions contribute to individual differences in health outcomes and psychological wellbeing $[13,14]$. A recent meta-analysis revealed that illness perceptions explained $21-30 \%$ of the variance of HRQoL across a range of illnesses [14]. Emotional responses to the disease and perceptions of consequences showed the strongest relationship with outcomes [14]. In a study specifically in COPD patients, perceptions of the consequences, symptoms and treatment control explained a higher percentage (56-59\%) of the variance in HRQoL [15]. Moreover, other studies in COPD patients showed that illness perceptions are associated with HRQoL [15-22]. COPD patients who have positive perceptions about the treatment possibilities, who believe that the impact of COPD on daily life is less serious and who are less emotionally disturbed by their disease experience better HRQoL [15-22].

Evidence highlights the importance of addressing patients' illness perceptions in order to influence illness-related behaviour and HRQoL in COPD patients [15, 20, 23, 24]. Based on the assumption that illness perceptions can be identified and modified [12, 13] and the evidence that changing these perceptions can lead to changes in behaviour, which could lead to better health outcomes [12, 13], we believe that illness perceptions should be addressed. However, specific guidelines how to discuss illness perceptions with COPD patients in clinical practice are lacking. Although there are studies evaluating the effectiveness of an illness perception intervention in patients who suffered a heart attack [25] and diabetes patients [26], protocols describing the interventions in detail are not available.

Therefore, we translated the existing evidence into the nurse-led COPD guidance, research on illness perception (COPD-GRIP) intervention [27, 28]. This tailor-made intervention starts with assessing and discussing illness perceptions, followed by improving patient's understanding of the relationship between perceptions and behaviour, creating an action plan and evaluating the actions $[27,28]$. The development and experiences of the nurses with the COPD-GRIP intervention are described elsewhere [28].

This cluster randomised trial aimed to assess whether the COPD-GRIP intervention is effective in improving health outcomes of COPD patients in primary care. The hypothesis was that COPD patients who receive the COPD-GRIP intervention would have better health outcomes in terms of health status, HRQoL, daily functioning and health-related behaviours.

\section{Methods}

Study design and participants

A two-arm, cluster randomised controlled trial with an intervention period of 6 weeks and follow-up period of 9 months was performed to test whether the nurse-led COPD-GRIP intervention leads to more improved health outcomes in COPD patients in primary care compared to usual nursing care (Netherlands Trial Register NTR 3945). A cluster consisted of a primary care practice or a home care service. 
Primary care practices $(n=34)$ and home care services $(n=6)$ were recruited throughout the Netherlands. A practice or home care service was eligible to participate in the study if a practice nurse or respiratory nurse provided consulting hours or home visits to guide COPD patients according to the standard COPD care as described in the Dutch COPD guidelines $[29,30]$. COPD patients were eligible if they were diagnosed by their general practitioner with mild to severe COPD, grades I to IV, according to the Global Initiative for Chronic Obstructive Lung Disease (GOLD) guidelines [4]. Other inclusion criteria were age $\geqslant 40$ years, a lung function test performed $\leqslant 1$ year prior to enrolment, physical and mental ability to complete the questionnaires and ability to understand and read the Dutch language. Patients were excluded if they had a life-threatening comorbid condition or if they had a primary diagnosis of asthma.

The medical research ethics committee (MREC) of the University Hospital Utrecht concluded that the Medical Research Involving Human Subjects Act (WMO) does not apply to this study; therefore, no WMO approval by the MREC was needed. All participants provided written informed consent to participate in the study.

\section{Setting}

Recruitment of primary care practices and home care services started in December 2012 and finished in October 2014. The inclusion of patients started in May 2013 and was closed in December 2014. The follow-up period ended in November 2015.

The practice nurses affiliated in the participating practices and home care services identified eligible COPD patients. Nurses approached all patients that were eligible according to the inclusion criteria.

Written informed consent was obtained from all patients before entry in the study.

The COPD-GRIP intervention was applied by the practice nurses within the context of three extra consultations within $\sim 6$ weeks. After three consultations the intervention was complete. The nurses in the control group were asked to continue their usual nursing care, based on the Dutch general practice COPD guidelines [30], in line with the GOLD guidelines [4].

\section{Intervention}

The COPD-GRIP intervention was applied individually for each participant and consisted of three extra face-to-face consultations, each lasting approximately half an hour [27, 28] (table 1). These consultations were planned within a $\sim 3$-week interval after inclusion in the study. The intervention has an equivalent structure for all patients. The specific content is individualised, based on a patient's responses and the needs of the patient.

During the first consultation, illness perceptions were assessed and discussed using the brief illness perception questionnaire (B-IPQ) [31]. In the second consultation, the patients' understanding of the relationship between their perceptions and their behaviour was discussed. Subsequently, patients were challenged to write an individualised care plan. In the last consultation, the actions the patients had taken to change their perceptions and behaviour were evaluated. An English version of the booklet describing the COPD-GRIP intervention can be found on our website (www.umcutrecht.nl/griponderzoek).

\section{TABLE 1 The COPD-GRIP (Chronic Obstructive Pulmonary Disease - Guidance, Research on Illness Perception) intervention}

The COPD-GRIP intervention is applied individually for each patient and consists of three face-to-face consultations, each lasting $~ 30$ min The three consultations are scheduled at $\sim 3$-week intervals

The specific content is individualised, based on the patient's questions, responses and the needs of the patient

First consultation: understanding the patient's illness perceptions

Second consultation: identifying the link between illness perceptions and behaviour

Third consultation: evaluating and discussing the individual care plan
1) Identifying and understanding patient's illness perceptions;

2) Assessing and discussing illness perceptions using the B-IPQ as a guide for tailoring the intervention

1) Identifying the link between illness perceptions and behaviour

2) Improving the patient's understanding of the relationship between their perceptions and their behaviour, by challenging them to draw up an individual care plan la short-term goal and a long-term goal, with strategies to achieve these)

1) Evaluating and discussing the individual care plan

2) Evaluating and assessing whether the individual care plan was successful and what new actions are necessary for the future

3) If the patient did not describe a care plan, discussing actions for the future

B-IPQ: Brief Illness Perception Questionnaire. 
The nurses working in the intervention practices were trained how to apply the COPD-GRIP intervention in a 4 -h educational session. During this session the theory that underpins the intervention was explained. Subsequently, the content of the booklet in which the intervention is described was discussed in detail [28]. The stages of the intervention were explained comprehensively and discussed step-by-step. A short animation movie was used to explain the content.

The first consultation of the intervention started shortly after each patient was included.

\section{Randomisation and masking}

Randomisation was performed at the level of the primary care practices/home care services before the inclusion of patients. A practice was randomised to the intervention group or control group using a computer-generated randomisation programme with block randomisation, developed by an independent data manager from the University Medical Center Utrecht.

Because of the nature of the intervention, participating nurses and patients could not be blinded to allocation.

\section{Treatment fidelity}

The following methods were used in order to support treatment fidelity. All nurses in the intervention groups were trained by the same trainer; furthermore, the intervention was described step-by-step in a booklet, which made it easier to use; during the study, all participating practice nurses were contacted by the study nurse monthly by telephone according to the protocol to discuss potential problems and register the number of consultations; finally, the practice nurses were asked to send the individualised care plans back to our centre.

\section{Outcomes}

All patient outcomes were collected by questionnaire and sent by post at baseline, 6 weeks, 3 months and 9 months. To prevent missing data, patients received one reminder to complete their questionnaires by means of a telephone call if they did not return the questionnaire within 3 weeks.

The primary outcome was health status on the Clinical COPD Questionnaire (CCQ) [32] at 9 months. The CCQ consists of 10 questions covering three domains: functional state, symptoms and mental state [32]. Secondary outcomes included HRQoL, as measured by the self-administered Chronic Respiratory Disease Questionnaire short form (CRQ-SAS), covering four dimensions: dyspnoea, fatigue, emotional function and mastery [33]. Other secondary outcomes were daily functioning, as measured by the Functional Performance Inventory (FPI) short form [34]. This questionnaire measures the extent to which people engage in their usual day-to-day activities to fulfil usual roles [34]. The B-IPQ [31] was used to asses illness perceptions concerning consequences, timeline, control, identity, concerns, understanding, emotional representations and causal representations. In addition, we measured impact of health education using the Health Education Impact Questionnaire (heiQ) [35]. This questionnaire was developed for the comprehensive evaluation of a patient education programme and covers eight independent dimensions such as positive and active engagement in life, health-directed behaviour, constructive attitudes and approaches, self-monitoring and insight. The Medical Research Council (MRC) dyspnoea score [36] was used to assess dyspnoea.

\section{Statistical analysis}

Sample size estimates were based on the mean difference in the total CCQ score (primary outcome) between intervention and control groups at 9 months. Sample size estimates for trials that randomise at the level of the individual were first used. Subsequently, to account for cluster randomisation a single inflation factor to the usual sample size was used in the power calculation [37]. The inflation factor is a function of cluster size $m$ and intraclass correlation $\rho: n^{*}=n(m) \times m=n[1+(m-1) \rho]$. The usual sample size of $\mathrm{n}=100$ per trial arm was based on the estimated effect size of 0.39 of the CCQ [38] with $\alpha=0.05$ and power was 0.80 [39]. Using the usual sample size, an upper estimated value of the intraclass correlation of $\rho=0.1$ [37] and the inflation factor, power calculations indicated that we needed 38 practice clusters with an average of 10 participants per cluster, leading to a total of 380 participants with a power of 0.80 and $\alpha=0.5$.

To compare demographic characteristics between the intervention and control group at baseline, a two-sample t-test $(\alpha=0.05)$ for normally distributed data and a Mann-Whitney test for non-normally distributed data were used.

The primary effectiveness analysis was an intention-to-treat analysis of the difference in mean CCQ score between groups at 9 months. Because of repeated measurements for all patients, we used multilevel repeated linear mixed modelling analyses [40]. 
We assessed the differences between the intervention and usual care group, the effects of measurement time points and whether group differences were dependent on measurement time points by defining a group-by-time interaction. If this group-by-time interaction is not significant, then the development of the outcome over time is not significant $(\alpha=0.05)$, meaning that there is no effect of the intervention. The primary explanatory parameters were the measured time points and the group allocation. These two parameters constitute the basic model. Other parameters (age, sex, MRC dyspnoea score, CRQ-SAS, FPI, B-IPQ and heiQ) were added to the model in order to see if the model improved. This analysis process was first conducted with the primary outcome HRQoL (CCQ). Subsequently, it was repeated with the secondary outcomes HRQoL (CRQ-SAS), daily activities (FPI), illness perceptions (B-IPQ) and health education impact (heiQ). The cluster was represented by a random intercept and a random slope model. The within-patient covariance was set as variance components.

An $a$ posteriori defined subgroup analysis was performed to identify whether dyspnoea (MRC dyspnoea score $\leqslant 2$ or $>2$ ) or sex were moderators.

All analyses were performed using SPSS for Windows (version 23.0; IBM, Armonk, NY, USA).

\section{Results}

30 primary care practices, five home care services and 204 patients were included in the study (figure 1). These patients met the inclusion criteria and gave their informed consent. However, five (2.5\%) patients did not return the baseline questionnaires ( $n=3$ in the intervention group and $n=2$ in the control group). Subsequently, the baseline analyses were performed with $n=199$ patients $(n=100$ in the intervention group and $n=99$ in the control group).

Dropout rates at 9 months were $17 \%$ in the intervention group and $14 \%$ in the usual-care group (figure 1). Patients who dropped out at 9 months had significantly worse scores on the CCQ, MRC, CRQ-SAS dyspnoea, CRQ-SAS fatigue, FPI, B-IPQ (consequences, concern and emotional response) questionnaires at baseline $(\mathrm{p}<0.05)$. Reasons given by patients for not participating in the study were the burden of filling in the follow-up questionnaires and the study could not be combined with work or family responsibilities.

The mean age was 68 years in the intervention group and 66 years in the usual-care group. The mean FEV1 (\% predicted) was similar in both study groups $(60.6 \%$ in the intervention group and $60.5 \%$ in the usual-care group). Most of the patients had moderate COPD $(n=61(62.2 \%)$ in the intervention group and $\mathrm{n}=63(63.6 \%)$ in the control group). There were no significant differences between the two groups at baseline, meaning that the two trial arms were well balanced on all variables at patient level (tables 2 and 3).

\section{COPD-GRIP group $\mathrm{n}=1 \mathrm{PCP}$ and $\mathrm{n}=1$ HCS did not include patients \\ $\mathrm{n}=3$ patients did not return baseline questionnaire}

\begin{tabular}{|l|}
\hline Dropout $n=18(17 \%)$ \\
No interest $n=4$ \\
Comorbidity $n=5$ \\
Died $n=3$ \\
Time $n=4$ \\
Other $n=2$ \\
\hline
\end{tabular}

Dropout $n=18(17 \%)$

Died $n=3$

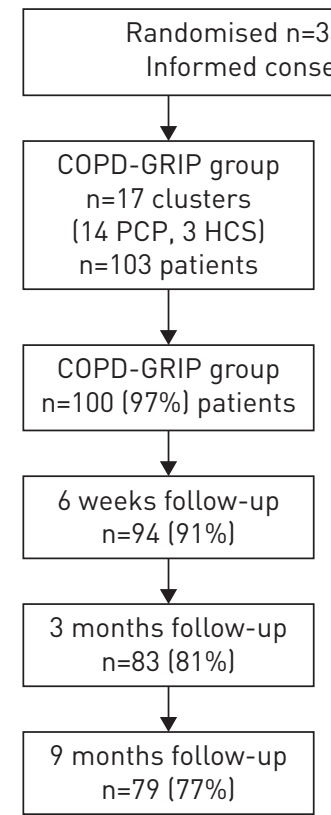

Randomised $n=34$ PCP and $n=6$ HCS

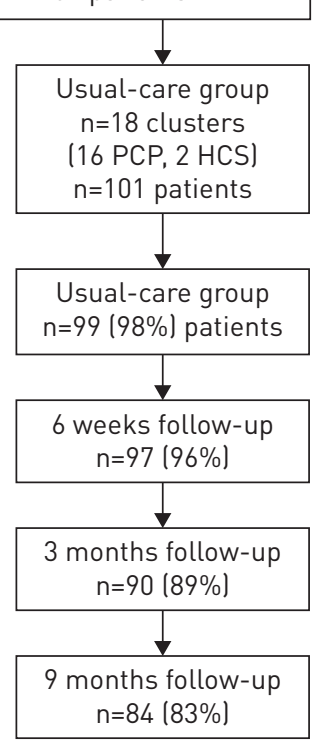

Usual-care group $n=3$ PCP did not include patients

$\mathrm{n}=2$ patients did not return baseline questionnaire

Dropout $n=14(14 \%)$ Too difficult $n=1$ No interest $n=3$ Died $n=2$

Time $n=5$

Other $n=3$

FIGURE 1 Flow chart of the study. PCP: primary care practice; HCS: home care service; COPD-GRIP: Chronic Obstructive Pulmonary Disease - Guidance, Research on Illness Perception. 


\begin{tabular}{|c|c|c|}
\hline & Intervention & Usual care \\
\hline Subjects & 100 & 99 \\
\hline Clusters & 17 & 18 \\
\hline Age years & $68.04 \pm 9.64$ & $65.78 \pm 9.61$ \\
\hline Male & $45(45.9)$ & 45 (45.4) \\
\hline FEV $1 \%$ predicted & $60.6 \pm 17.6$ & $60.5 \pm 20.1$ \\
\hline \multicolumn{3}{|l|}{ GOLD stage $\#$} \\
\hline I (mild) & $9(9.2)$ & $9(9.0)$ \\
\hline II (moderate) & $61(62.2)$ & $63(63.6)$ \\
\hline III (severe) & $23(23.5)$ & $18(18.2)$ \\
\hline IV (very severe) & $5(5.1)$ & $9(9.1)$ \\
\hline \multicolumn{3}{|l|}{ Education level } \\
\hline Lower secondary or less & 70 (71.4) & $65(65.6)$ \\
\hline Upper secondary & $18(18.4)$ & $21(21.2)$ \\
\hline College/university & $10(10.2)$ & 8 (8.1) \\
\hline Current smoker & $27(27.6)$ & 32 (32.3) \\
\hline Living alone & $44(44.9)$ & $39(39.4)$ \\
\hline \multicolumn{3}{|c|}{ Contact with healthcare professional ๆ } \\
\hline Nurse & 268 & 195 \\
\hline GP & 323 & 263 \\
\hline Physiotherapist & 414 & 360 \\
\hline Dietician & 6 & 33 \\
\hline Increase of complaints ${ }^{+}$ & 248 (30.3) & $177(22.3)$ \\
\hline Hospital admissions ${ }^{\S}$ & 13 & 11 \\
\hline \multicolumn{3}{|l|}{ Comorbidities } \\
\hline Myocardial infarction & $9(9.1)$ & $6(6.1)$ \\
\hline Heart failure & $11(11.1)$ & $4(4.1)$ \\
\hline Cerebrovascular accident & $6(6.1)$ & $3(3.1)$ \\
\hline Diabetes & $5(5.1)$ & $21(21.1)$ \\
\hline \multicolumn{3}{|c|}{$\begin{array}{l}\text { Data are presented as } \mathrm{n} \text {, mean } \pm \text { SD or } \mathrm{n}(\%) \text {. FEV } 1 \text { : forced expiratory volume in } 1 \mathrm{~s} \text {; GOLD: Global Initiative } \\
\text { for Chronic Obstructive Lung Disease; GP: general practitioner. \#: I (mild): FEV } 1 \geqslant 80 \% \text { pred; II (moderate): } \\
\text { FEV } 1 \geqslant 50 \% \text { to }<80 \% \text { pred; III (severe): FEV } 1 \geqslant 30 \% \text { to }<50 \% \text { pred; IV (very severe): FEV } 1<30 \% \text { pred; }{ }^{\text {I }} \text { : total } \\
\text { counts of contacts during study period (intervention period of } 6 \text { weeks and } 9-\text { month follow-up); }{ }^{+} \text {total to } \\
\text { counts of reported increase of complaints during study period (intervention period of } 6 \text { weeks and } \\
9 \text {-month follow-up); } \S \text { : total counts of reported hospital admissions during study period (intervention } \\
\text { period of } 6 \text { weeks and 9-month follow-up). }\end{array}$} \\
\hline
\end{tabular}

\section{Primary outcome}

No statistically significant differences in the CCQ were detected between the groups at week 6 or after 3 and 9 months (table 4). Furthermore, the group*time interaction with the outcome CCQ was not significant $(\mathrm{p}=0.197)$, meaning that the COPD-GRIP intervention could not improve health status over time (figure 2).

\section{Secondary outcomes and subgroup analyses}

No statistically significant differences were detected between groups in the secondary outcomes HRQoL, as measured by the CRQ-SAS ( $\mathrm{p}=0.162-0.631$ ), and daily activities, as measured by the FPI ( $\mathrm{p}=0.074$; table 4).

The analyses showed a statistically significant treatment effect on the health-directed behaviour domain of the heiQ at 6 weeks $(\mathrm{p}=0.024)$, as shown in table 4 . This means that the COPD-GRIP intervention improved health behaviour shortly after the intervention, but this effect was not preserved over time (figure 3 ).

Furthermore, there was a statistically significant treatment effect on the personal control domain of the B-IPQ ( $p=0.005$ at 9 months), meaning that the COPD-GRIP intervention improved the perception of the ability to control the disease. However, at 3 months there was a decrease of the perception of control (figure 4), meaning that this result is clinically difficult to interpret.

An $a$ posteriori defined subgroup analysis to identify whether dyspnoea (MRC dyspnoea score $\leqslant 2$ or $>2$ ) or sex are moderators showed no significant effect of the intervention (table 5).

Analysis using the minimal clinical important differences (MCID) of 0.4 for the CCQ [38] revealed that $15.7 \%(n=16)$ of the patients in the intervention group and $12.9 \%(n=13)$ of the patients in the control 


\begin{tabular}{|c|c|c|c|}
\hline & Intervention & Ususal care & Reference range \\
\hline $\mathrm{CCQ}^{\#}$ & $1.9 \pm 1.2$ & $1.6 \pm 1.1$ & $0-6$ \\
\hline Symptoms & $2.2 \pm 1.2$ & $2.0 \pm 1.1$ & \\
\hline Functional state & $2.0 \pm 1.5$ & $1.7 \pm 1.4$ & \\
\hline Mental state & $1.0 \pm 1.3$ & $0.9 \pm 1.1$ & \\
\hline \multicolumn{4}{|l|}{ B-IPQ } \\
\hline Consequences & $5.5 \pm 2.9$ & $4.9 \pm 2.8$ & $0.0-10.0$ \\
\hline Timeline & $9.5 \pm 1.6$ & $9.6 \pm 1.7$ & $0.0-10.0$ \\
\hline Personal control & $5.5 \pm 2.7$ & $6.4 \pm 2.3$ & $0.0-10.0$ \\
\hline Treatment control & $7.0 \pm 2.6$ & $7.0 \pm 2.0$ & $0.0-10.0$ \\
\hline Identity & $5.4 \pm 2.5$ & $4.9 \pm 2.7$ & $0.0-10.0$ \\
\hline Concern & $5.0 \pm 3.0$ & $5.4 \pm 3.1$ & $0.0-10.0$ \\
\hline Comprehensibility & $7.6 \pm 2.4$ & $7.1 \pm 2.6$ & $0.0-10.0$ \\
\hline Emotional response & $3.9 \pm 3.2$ & $3.6 \pm 2.9$ & $0.0-10.0$ \\
\hline $\mathrm{MRC}^{+}$ & $2.2 \pm 1.3$ & $1.9 \pm 1.4$ & $0-5$ \\
\hline \multicolumn{4}{|l|}{ CRQ-SAS ${ }^{\S}$} \\
\hline Dyspnoea & $5.8 \pm 1.4$ & $5.6 \pm 1.5$ & $1-8$ \\
\hline Fatigue & $4.5 \pm 1.4$ & $4.7 \pm 1.4$ & $1-7$ \\
\hline Emotional & $5.0 \pm 1.2$ & $5.2 \pm 1.2$ & $1-7$ \\
\hline Mastery & $5.4 \pm 1.2$ & $5.4 \pm 1.3$ & $1-7$ \\
\hline $\mathrm{FPI}^{\S}$ & $1.7 \pm 0.7$ & $1.8 \pm 0.6$ & $1-3$ \\
\hline \multicolumn{4}{|l|}{ heiQ $Q^{\S}$} \\
\hline Health-directed behaviour & $2.9 \pm 0.7$ & $3.1 \pm 0.7$ & $1-4$ \\
\hline Positive and active engagement in life & $3.0 \pm 0.6$ & $3.1 \pm 0.5$ & $1-4$ \\
\hline Emotional distress & $3.1 \pm 0.7$ & $3.1 \pm 0.7$ & $1-4$ \\
\hline Self-monitoring and insight & $3.1 \pm 0.4$ & $3.1 \pm 0.4$ & $1-4$ \\
\hline Constructive attitudes and approaches & $3.2 \pm 0.6$ & $3.3 \pm 0.5$ & $1-4$ \\
\hline Skill and technique acquisition & $3.0 \pm 0.4$ & $3.0 \pm 0.5$ & $1-4$ \\
\hline Social integration and support & $3.0 \pm 0.6$ & $3.0 \pm 0.6$ & $1-4$ \\
\hline Health service navigation & $3.2 \pm 0.4$ & $3.2 \pm 0.4$ & $1-4$ \\
\hline \multicolumn{4}{|c|}{$\begin{array}{l}\text { Data are presented as mean } \pm \text { SD or } n \text {. CCQ: Clinical COPD Questionnaire; B-IPQ: Brief Illness Perception } \\
\text { Questionnaire; MRC: Medical Research Council dyspnoea score; CRQ-SAS: self-administered Chronic } \\
\text { Respiratory Disease Questionnaire short form; FPI: Functional Performance Inventory; heiQ: Health } \\
\text { Education Impact Questionnaire. \#: lower scores mean better health-related quality of life (HRQoL); } \\
\text { १: lower scores on consequences, timeline, identity, concern and emotional response and higher scores on } \\
\text { personal control, treatment control and comprehensibility mean more positive perceptions; }{ }^{+} \text {: lower } \\
\text { scores mean less burden of dyspnoea; }{ }^{\S} \text { : higher scores mean better HRQoL, functional performance and } \\
\text { health education impact. }\end{array}$} \\
\hline
\end{tabular}

group had a MCID $\geqslant-0.4$, meaning that these patients experienced a better HRQoL compared to baseline. The difference between the groups was not significant.

\section{Discussion}

This cluster randomised trial revealed that the COPD-GRIP intervention, which focuses on identifying, discussing and evaluating illness perceptions could not improve health status (measured using the CCQ) and HRQoL (measured using the CRQ-SAS) in COPD patients in primary care in the Netherlands. The intervention did demonstrate advantages on the perception of the ability to control the disease and on health-related behaviours in the short term. However, these gains did not persist in the long term.

While the intervention is based on the evidence regarding illness perceptions, health outcomes $[15,17,20,21]$, recent developments concerning patient-centred care $[41,42]$ and was applied in real nursing practice, we could not show benefits in health status and HRQoL in COPD patients.

There are some considerations for not identifying these benefits. Firstly, it could be that the time period of the intervention was too short. In order to achieve more comprehensive guidance and results in the long term, some ongoing support by the nurses and an extension of the follow-up period of the intervention could be needed. Recent evidence from an individual patient data meta-analysis concerning characteristics of self-management interventions in COPD patients shows that there are risk reductions for each increasing contact and each increasing month of duration of a self-management intervention [43]. A Cochrane review [42] shows that the effects of personalised care planning were greater when more stages 
TABLE 4 Clinical outcomes, corrected for clustering, time, treatment and Medical Research Council dyspnoea score ${ }^{\#}$ for the COPD-GRIP (Chronic Obstructive Pulmonary Disease - Guidance, Research on Illness Perception) intervention group and the usual-care group

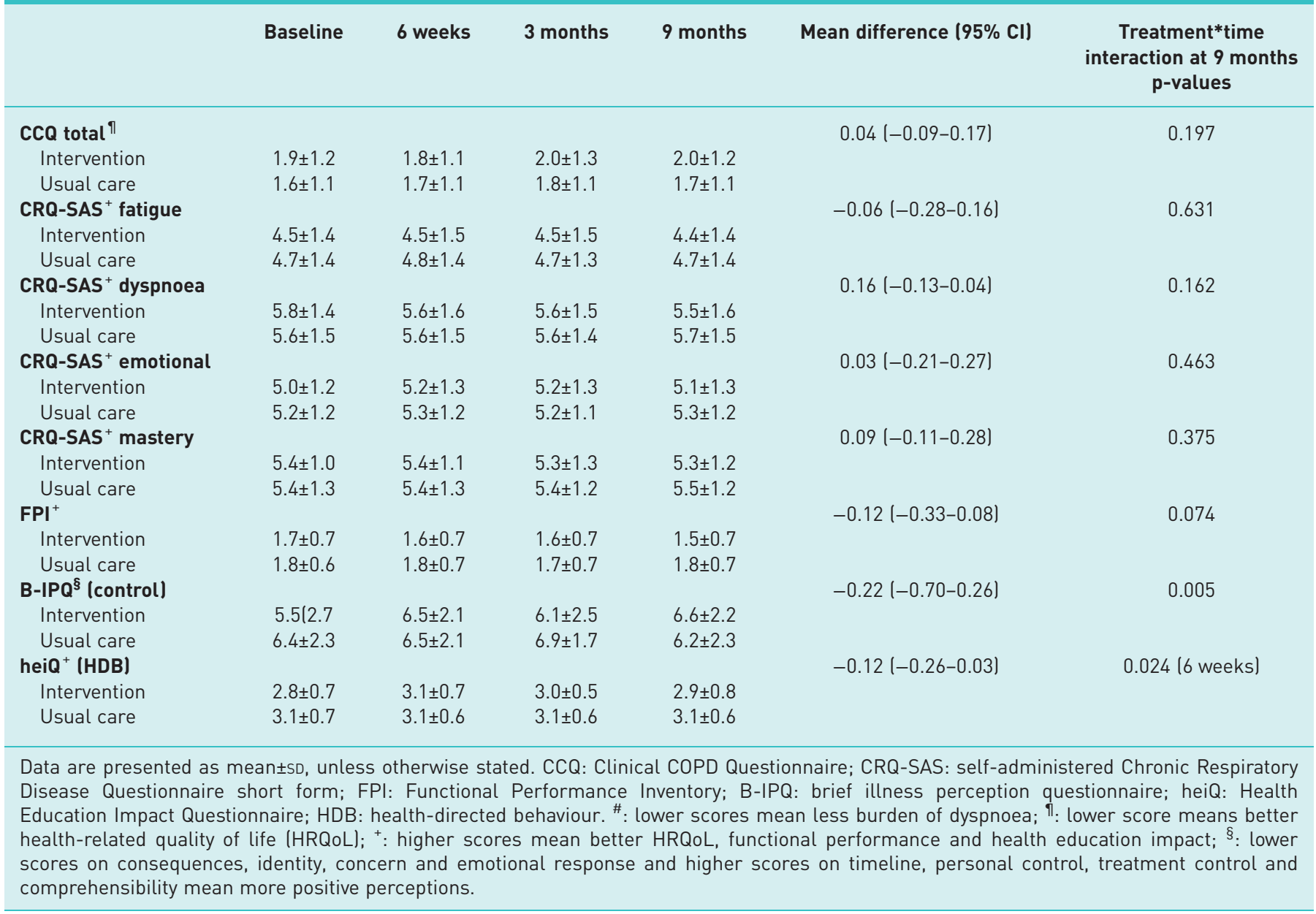

of the care planning cycle were completed, and when contacts between patients and health professionals were more frequent.

Secondly, we know from our study concerning the exploration of facilitators and barriers of the COPD-GRIP intervention [28] that incorporating the intervention in real nursing practice should have been supported by more intensive training on the job for the nurses, to identify more benefits. Although the nurses perceived the intervention as valuable and feasible, the study revealed that the nurses would like to receive more training in applying the intervention in the future [28].

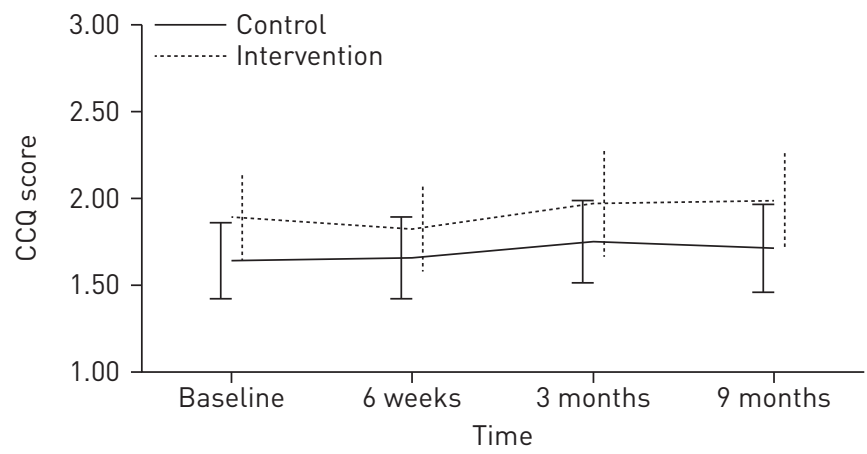

FIGURE 2 Clinical COPD Questionnaire (CCQ) scores over time. Data are presented as mean (95\% CI). 


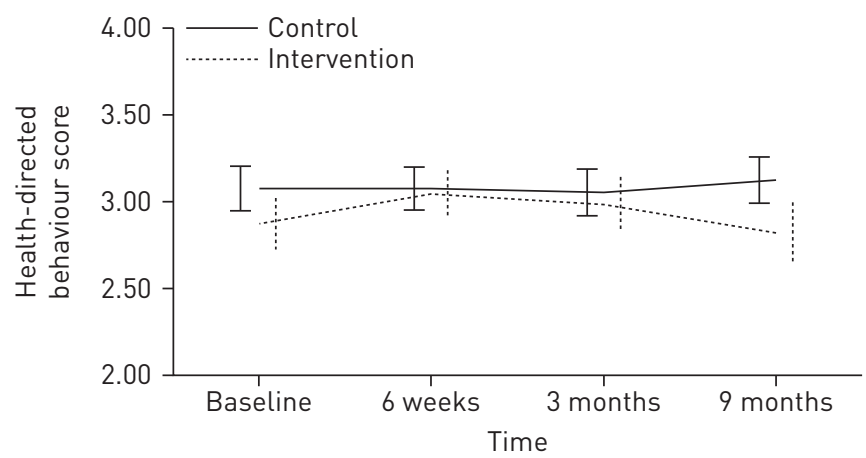

FIGURE 3 Mean health-directed behaviour scores over time. Data are presented as mean $(95 \% \mathrm{CI})$.

Thirdly, given the fact that most of the participants had a lower educational level, it could be possible that the intervention was too difficult for them and therefore it could be questioned whether the intervention is appropriate for every patient.

Fourthly, although the CCQ is a highly recommended outcome measure in COPD care and research [44], it could be questioned whether the CCQ is the best possible outcome measure to capture the perceived benefits of the COPD-GRIP intervention that might be achieved by the individual patient. The CCQ measures health status within three domains: symptoms, functional state and mental state. Because the impact of COPD on daily life varies among individuals, and symptoms may change over time as the disease progresses, it could be questioned whether more individualised multidimensional response measures, including domains such as adaptability, adjustment to disease and resilience should be more appropriate. A possible solution could be the use of computerised adaptive testing (CAT) [45]. This method allows more flexibility because the selection of questions depends on a patient's response to previous items [45]. A CAT for assessing HRQoL in COPD patients has been developed by PAAP et al. [46].

Of particular relevance to this discussion is the recent debate concerning multidimensional measures in COPD by Spruit et al. [47] and Ambrosino and Clini [48] who raise the urgent need to develop measures in COPD research that take into account the large heterogeneity of clinical manifestations and individual differences in COPD to do justice to individual responses to therapies.

Our study revealed that the intervention significantly improved health behaviours (measured by the heiQ) in the short term; however, this improvement was not substantial. An effect size of 0.5 or a standard deviation of 0.5 is defined as substantial, and our study showed an effect of 0.3 . This small improvement means that some patients changed aspects of their health behaviours. These activities may include changes in diet, exercise and relaxation routines [35]. Although it is not known which specific aspect of the intervention caused this effect, our method and analyses allow us to draw these conclusions. It could be possible that this short-term effect was caused by the extra attention of the nurse, or a better understanding of the relationship between perceptions and behaviour, which could have resulted in a short-term behaviour change.

Furthermore, we did find an effect on perceived ability to control the disease. However, this result is difficult to interpret. The perception of disease control improved at 6 weeks, declined between 6 weeks and

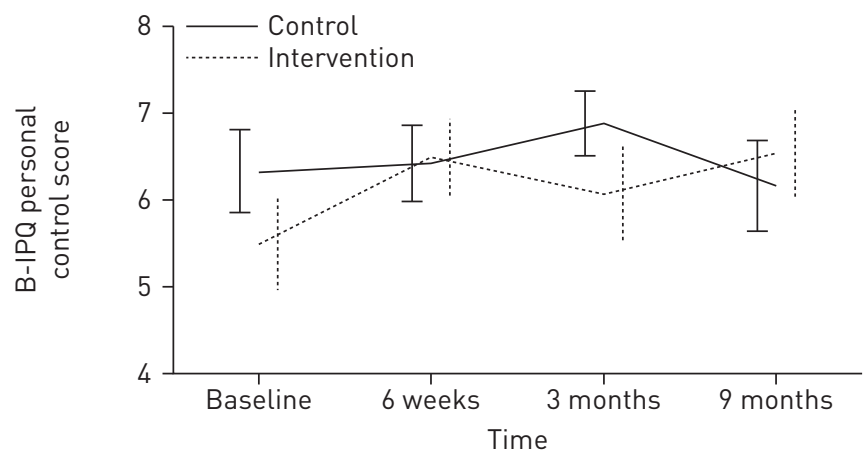

FIGURE 4 Mean Brief Illness Perception Questionnaire (B-IPQ) personal control scores over time. Data are presented as mean $(95 \% \mathrm{CI})$ 


\begin{tabular}{|c|c|c|c|c|c|c|}
\hline & Subjects n & Baseline & 6 weeks & 3 months & 9 months & $\begin{array}{c}\text { Treatment*time } \\
\text { interaction } \\
\text { p-values }\end{array}$ \\
\hline MRC score $\leqslant 2$ & & & & & & 0.595 \\
\hline Intervention & 64 & $1.3 \pm 0.7$ & $1.3 \pm 0.7$ & $1.3 \pm 0.9$ & $1.5 \pm 0.8$ & \\
\hline Usual care & 68 & $1.2 \pm 0.8$ & $1.2 \pm 0.8$ & $1.3 \pm 0.8$ & $1.3 \pm 0.7$ & \\
\hline MRC score >2 & & & & & & 0.165 \\
\hline Intervention & 20 & $3.0 \pm 1.1$ & $3.0 \pm 1.1$ & $3.2 \pm 1.1$ & $3.2 \pm 1.1$ & \\
\hline Usual care & 23 & $2.7 \pm 0.9$ & $2.9 \pm 1.0$ & $3.0 \pm 0.9$ & $3.0 \pm 1.0$ & \\
\hline Male & & & & & & 0.811 \\
\hline Intervention & 33 & $1.8 \pm 1.3$ & $1.4 \pm 0.9$ & $1.8 \pm 1.3$ & $1.7 \pm 1.0$ & \\
\hline Usual care & 41 & $1.7 \pm 1.0$ & $1.9 \pm 1.1$ & $1.8 \pm 1.1$ & $1.8 \pm 1.3$ & \\
\hline Female & & & & & & 0.126 \\
\hline Intervention & 51 & $2.2 \pm 1.3$ & $2.1 \pm 1.2$ & $2.3 \pm 1.4$ & $2.2 \pm 1.3$ & \\
\hline Usual care & 53 & $1.6 \pm 1.1$ & $1.6 \pm 1.2$ & $1.8 \pm 1.1$ & $1.6 \pm 1.0$ & \\
\hline
\end{tabular}

3 months, and at 9 months a small improvement was found. These results show that applying the COPD-GRIP intervention resulted in a higher experienced sense of control directly after the intervention. However, without guidance over time there was no stable improvement. Furthermore, we did not find the same effect on the comparable item heiQ (constructive attitudes and approaches), therefore these results must be addressed with caution. However, we could expect some kind of changes in the control dimension because some people changed their behaviour, which could lead to a subjective sense of control.

Although this trial could not show significant effects on HRQoL, the COPD-GRIP intervention did show some positive findings. During the trial two qualitative studies were performed, in order to identify the experiences of the patients and nurses with the COPD-GRIP intervention [28]. These studies showed various benefits. Patients in the intervention group expressed that they were more aware of the consequences of COPD and they were willing to undertake a more active lifestyle (unpublished data). Simultaneously, the nurses described the intervention as a very useful tool to provide care that is tailored to patients' needs. The COPD-GRIP intervention provides nurses with a structured way to address illness perceptions in daily clinical care in order to understand the behaviour of the patient [28]. The qualitative studies show that the COPD-GRIP intervention improved the communication and interaction between patient and nurse. Given the complex and lifelong nature of COPD, these improvements are relevant.

In the light of the findings of this trial and the positive experiences of the patients as well as the nurses regarding the intervention, it seems reasonable to rethink the process of the intervention by expanding the time period of the intervention and providing intensive training on the job for nurses. This means that they could answer questions and get instructions during their work, including discussion sessions where nurses could share their experiences and learn from colleagues.

Whereas in other patient groups, such as patients recovering from a heart attack [25] and poorly controlled type 2 diabetes patients [26] an illness perception intervention improved health-related outcomes, we could not draw comparable conclusions. PETRIE et al. [25] conducted an intervention in heart attack patients at a very early stage after a heart attack and prior to hospital discharge and KEOGH et al. [26] studied a family-based intervention in poorly controlled diabetes patients aiming to reach glycaemic control targets. However, there are some important differences between our study and these studies. Firstly, the heart attack patient group is not comparable with the COPD patients. Whereas heart attack patients were confronted with a life-threatening acute disease, COPD patients have a mostly slow progressive disease. Secondly, the outcome measures are not comparable. PETRIE et al. [25] used recovery and return to work as an outcome measure and KeOGH et al. [26] used glycated haemoglobin in addition to psychological wellbeing and beliefs. In addition, a large cluster randomised trial, undertaken in COPD patients in primary care in the Netherlands assessing the effectiveness of integrated disease management on HRQoL could not show an improvement on the outcome (CCQ) [49]. More or less the same pattern of change in health status improvements in the first follow-up period, but no preservation over time was revealed in a large medication study in COPD patients [50]. These studies show some similarities in the pattern of responses of COPD patients. These results raise the question of what can be done to improve 
HRQoL in a complex disease such as COPD and to sustain health status improvements after initial treatment. Although improving HRQoL in complex diseases such as COPD is not easy, a vital aspect of care is recognising and acknowledging individual differences. A starting point is exploring the perceptions of patients and assessing their needs as described by themselves.

\section{Strengths and limitations of this study}

To best of our knowledge, this is the first study assessing the effect of an individualised illness perception intervention in COPD patients in primary care. Strengths of this study include embedding the clinical trial in regular nursing practice and the inclusion of a wide range of patient outcome measures relevant for primary-care nursing. Another strength is the operationalisation of the crucial, but rather neglected theoretical concept of illness perceptions within patient-centred care by identifying, discussing and evaluating illness perceptions. Additionally, we applied sophisticated multilevel and longitudinal analyses to assess the effectiveness of the intervention.

Despite our expectations and effort, we did not succeed in including the number of patients calculated in the power calculation, which is a limitation of this study. Recruitment of patients was performed by the practice nurses by means of professional invitation. Despite the commitment of the general practitioners and the practice nurses, the heavy workload for practice nurses in primary care may have contributed to a suboptimal recruitment of patients. Subsequently, it could be possible that we missed a real effect by including insufficient numbers. Dropouts after 9 months were low; however, their scores at baseline were significantly worse. This could raise questions about generalisability. However, incorporating baseline scores in the analyses did not result in treatment effects, indicating that it is unlikely that dropout rates biased the results. Furthermore, we have no information about the patients who did not take part in the trial; in other words, we cannot identify characteristics of nonresponders. Subsequently, we could not analyse whether these characteristics could influence the effect of the study.

Another limitation is the lack of a detailed description of the treatment fidelity in this study. Although we used a standardised approach in the educational sessions and the protocol of the intervention, there is no insight into the exact content and nature of the consultations. As a consequence, it is not known if the nurses applied the intervention as it was described in the booklet. However, all patients within the intervention group $(n=100)$ received the additional consultations. These patients were challenged (not forced) to draw up an individualised care plan. Finally, we received 83 out of 100 individualised care plans back, meaning that a large number of patients within the intervention group described a care plan together with the nurses, as recommended in the intervention tool.

\section{Conclusion}

The COPD-GRIP intervention, practiced by nurses, could not improve health status and HRQoL in COPD patients in primary care in the Netherlands. The intervention does influence the ability to control the disease and health-directed behaviour in the short term. Therefore, taking illness perceptions into account when stimulating healthy behaviours in COPD patients should be considered. Further study on illness perceptions influencing health status and HRQoL is needed.

\section{Acknowledgements}

We would like to thank Nicole Boekema (Julius Center for Health Sciences and Primary Care, University Medical Center Utrecht, Utrecht, the Netherlands) for developing a computer-generated randomisation programme. We would like to thank all the nurses and patients within the COPD-GRIP trial who gave their time to participate in this study. We would like to thank Simone Sluis and Marieke Zwakman (Dept of Respiratory Diseases, Division of Heart and Lungs, University Medical Center Utrecht) for their valuable help in the organisation of the trial.

\section{References}

1 Decramer M, Janssens W. Chronic obstructive pulmonary disease and comorbidities. Lancet Respir Med 2013; 1: 73-83.

2 Vos T, Barber RM, Bell B, et al. Global, regional, and national incidence, prevalence, and years lived with disability for 301 acute and chronic diseases and injuries in 188 countries, 1990-2013: a systematic analysis for the Global Burden of Disease study 2013. Lancet 2015; 386: 743-800.

3 López-Campos JL, Tan W, Soriano JB. Global burden of COPD. Respirology 2016; 21: 14-23.

4 Global Initiative for Chronic Obstructive Lung Disease (GOLD). Global Strategy for the Diagnosis, Management and Prevention of COPD 2015. Date last accessed: January 2016. Date last updated: January 2015.

5 Fletcher MJ, Dahl BH. Expanding nurse practice in COPD: is it key to providing high quality, effective and safe patient care? Prim Care Respir J 2013; 22: 230-233.

6 Myers TR. Thinking outside the box: moving the respiratory care profession beyond the hospital walls. Respir Care 2013; 58: 1377-1385.

7 McDonald R, Campbell S, Lester H. Practice nurses and the effects of the new general practitioner contract in the English National Health Service: the extension of a professional project? Soc Sci Med 2009; 68: 1206-1212. 
Schermer T, van Weel C, Barten F, et al. Prevention and management of chronic obstructive pulmonary disease (COPD) in primary care: position paper of the European Forum for Primary Care. Qual Prim Care 2008; 16: 363-377.

9 Freund T, Everett C, Griffiths P, et al. Skill mix, roles and remuneration in the primary care workforce: who are the healthcare professionals in the primary care teams across the world? Int J Nurs Stud 2015; 52: 727-743.

10 Agusti A, Calverley PM, Celli B, et al. Characterisation of COPD heterogeneity in the ECLIPSE cohort. Respir Res 2010; 11: 122 .

11 Leventhal HL, Brissette I, Leventhal EA. Common-sense model of self-regulation of health and illness. In Cameron LD, Leventhal H, eds. The Self-regulation of Health and Illness Behaviour. London, Routledge, 2003; pp. $42-65$.

12 Leventhal H, Phillips LA, Burns E. The common-sense model of self-regulation (CSM): a dynamic framework for understanding illness self-management. J Behav Med 2016; 39: 935-946.

13 Hagger MS, Orbell S. A meta-analytic review of the common-sense model of illness representations. Psychol Health 2003; 18: 141-184.

14 Dempster M, Howell D, McCorry NK. Illness perceptions and coping in physical health conditions: a meta-analysis. J Psychosom Res 2015; 79: 506-513.

15 Weldam SW, Lammers JW, Heijmans MJ, et al. Perceived quality of life in chronic obstructive pulmonary disease patients: a cross-sectional study in primary care on the role of illness perceptions. BMC Fam Pract 2014; 15: 140

16 Bonsaksen T, Haukeland-Parker S, Lerdal A, et al. A 1-year follow-up study exploring the associations between perception of illness and health-related quality of life in persons with chronic obstructive pulmonary disease. Int $J$ Chron Obstruct Pulmon Dis 2014; 9: 41-50.

17 Scharloo M, Kaptein AA, Schlösser M, et al. Illness perceptions and quality of life in patients with chronic obstructive pulmonary disease. J Asthma 2007; 44: 575-581.

18 Weldam S, Lammers J, Decates R, et al. Daily activities and health-related quality of life in patients with chronic obstructive pulmonary disease: psychological determinants: a cross-sectional study. Health Qual Life Outcomes 2013; 11: 190.

19 Kaptein AA, Scharloo M, Fischer MJ, et al. Illness perceptions and COPD: an emerging field for COPD patient management. J Asthma 2008; 45: 625-629.

20 Mewes R, Rief W, Kenn K, et al. Psychological predictors for health-related quality of life and disability in persons with chronic obstructive pulmonary disease (COPD). Psychol Health 2016; 31: 470-486.

21 Vaske I, Kenn K, Keil DC, et al. Illness perceptions and coping with disease in chronic obstructive pulmonary disease: effects on health-related quality of life. J Health Psychol 2017; 22: 1570-1581.

22 Zoeckler N, Kenn K, Kuehl K, et al. Illness perceptions predict exercise capacity and psychological well-being after pulmonary rehabilitation in COPD patients. J Psychosom Res 2014; 76: 146-151.

23 Miravitlles M, Ferrer J, Baró E, et al. Differences between physician and patient in the perception of symptoms and their severity in COPD. Respir Med 2013; 107: 1977-1985.

24 Heijmans M, Foets M, Rijken M, et al. Stress in chronic disease: do the perceptions of patients and their genera practitioners match? Br J Health Psychol 2001; 6: 229-242.

25 Petrie KJ, Cameron LD, Ellis CJ, et al. Changing illness perceptions after myocardial infarction: an early intervention randomized controlled trial. Psychosom Med 2002; 64: 580-586.

26 Keogh KM, Smith SM, White P, et al. Psychological family intervention for poorly controlled type 2 diabetes. Am J Manag Care 2011; 17: 105-113.

27 Weldam SWM, Lammers JWJ, Schuurmans MJ. COPD-GRIP Intervention. Date last accessed: May 29, 2015. Date last updated: 2015.

28 Weldam SWM, Lammers JW, Zwakman M, et al. Nurses' perspectives of a new individualized nursing care intervention for COPD patients in primary care settings: a mixed method study. Appl Nurs Res 2017; 33: 85-92.

29 Long Alliantie Nederland (LAN). Zorgstandaard COPD. [COPD Care Standards.] www.longalliantie.nl/ zorgstandaard-copd Date last accessed: February 2017. Date last updated: 2016.

30 Smeele I, van Weel C, Van Schayck CP, et al. NHG standaard COPD. [NHG COPD Standards.] Huisarts Wetenschap 2007; 50: 362-379.

31 Broadbent E, Petrie KJ, Main J, et al. The brief illness perception questionnaire. J Psychosom Res 2006; 60: 631-637.

32 van der Molen T, Willemse B, Schokker S, et al. Development, validity and responsiveness of the clinical COPD questionnaire. Health Qual Life Outcomes 2003; 1: 13.

33 Williams JE, Singh SJ, Sewell L, et al. Development of a self-reported chronic respiratory questionnaire (CRQ-SR). Thorax 2001; 56: 954-959.

34 Leidy NK, Knebel A. In search of parsimony: reliability and validity of the Functional Performance Inventory-Short Form. Int J Chron Obstruct Pulmon Dis 2010; 5: 415-423.

35 Osborne RH, Elsworth GR, Whitfield K. The Health Education Impact Questionnaire (heiQ): an outcomes and evaluation measure for patient education and self-management interventions for people with chronic conditions. Patient Educ Couns 2007; 66: 192-201.

36 Bestall JC, Paul EA, Garrod R, et al. Usefulness of the Medical Research Council (MRC) dyspnoea scale as a measure of disability in patients with chronic obstructive pulmonary disease. Thorax 1999; 54: 581-586.

37 Friedman LK, Furberg CD, DeMets DL. Fundamentals of Clinical Trials. New York, Springer-Verlag New York, 1998.

38 Kocks JW, Tuinenga MG, Uil SM, et al. Health status measurement in COPD: the minimal clinically important difference of the clinical COPD questionnaire. Respir Res 2006; 7: 62.

39 Polit DF, Beck CD. Nursing Research, Generating and Assessing Evidence for Nursing Research. 8th Edn Lippincott Williams and Wilkins, 2007; p. 816.

40 Heck RH, Thomas SJ, Tabata LN. Multilevel and Longitudinal Modeling with IBM SPSS. 2nd Edn. New York, Routledge Taylor \& Francis Group, 2014.

41 Rice K, Bourbeau J, MacDonald R, et al. Collaborative self-management and behavioral change. Clin Chest Med 2014; 35: 337-351. 
42 Coulter A, Entwistle VA, Eccles A, et al. Personalised care planning for adults with chronic or long-term health conditions. Cochrane Database Syst Rev 2015; 3: CD010523.

43 Jonkman NH, Westland $\mathrm{H}$, Trappenburg JCA, et al. Characteristics of effective self-management interventions in patients with COPD: individual patient data meta-analysis. Eur Respir J 2016; 48: 55-68.

44 Cave AJ, Atkinson L, Tsiligianni IG, et al. Assessment of COPD wellness tools for use in primary care: an IPCRG initiative. Int J Chron Obstruct Pulmon Dis 2012; 7: 447-456.

45 Wainer H. Computerized Adaptive Testing. 2nd Edn. New York, Routledge Taylor and Francis Group, 2000.

46 Paap MC, Lenferink LI, Herzog N, et al. The COPD-SIB: a newly developed disease-specific item bank to measure health-related quality of life in patients with chronic obstructive pulmonary disease. Health Qual Life Outcomes 2016; 14: 97.

47 Spruit MA, Franssen FME, Rutten EPA, et al. A new perspective on COPD exacerbations: monitoring impact by measuring physical, psychological and social resilience. Eur Respir J 2016; 47: 1024-1027.

48 Ambrosino N, Clini EM. Response to pulmonary rehabilitation: toward personalised programmes? Eur Respir J 2015; 46: 1538-1540.

49 Kruis AL, Boland MR, Assendelft WJ, et al. Effectiveness of integrated disease management for primary care chronic obstructive pulmonary disease patients: results of cluster randomised trial. BMJ 2014; 349: g5392.

50 Jones P, Anderson J, Calverley P, et al. Health status in the TORCH study of COPD: treatment efficacy and other determinants of change. Respir Res 2011; 12: 71. 